\title{
1.9 picosecond high-sensitivity sampling optical temporal analyzer
}

\author{
Yi Chen, a) Steve Williamson, ${ }^{\text {b) }}$ and Tim Brock \\ Ultrafast Science Laboratory, University of Michigan, Ann Arbor, Michigan 48109-2099
}

(Received 1 September 1993; accepted for publication 11 November 1993)

\begin{abstract}
An all solid-state, high-sensitivity sampling optical temporal analyzer has been demonstrated by integrating two interdigitated picosecond metal-semiconductor-metal photodelectors into a coplanar transmission line structure. The full width at half-maximum of the response time is $1.9 \mathrm{ps}$. The noise equivalent optical power is $500 \mathrm{pW}$, and the dynamic range is more than $60 \mathrm{~dB}$.
\end{abstract}

The rapid development of ultrafast sciences places an increasing demand on high-spccd, high-dynamic range measurement techniques. An optical correlation technique based on photoconductivity for measuring ultrafast laser pulses was first demonstrated by Austin et al. ${ }^{1}$ However, due to relatively low efficiency of both signal detection and sampling, such a system was not able to measure weak optical signals. Recently, Li et al. ${ }^{2}$ showed that a Schottky photodiode with the integration of a microwave detector can measure weak optical laser pulses with picosecond resolution. In this letter, we report an all solid-state circuit that we believe is the best in terms of speed and dynamic range for performing optical correlation measurements. Our device structure is based on Ketchen et al.' $\mathrm{s}^{3}$ original work with incorporation of a highspeed, high-responsivity metal-semiconductor-metal (MSM) interdigitated photodetector/switch developed in our laboratory. As the result, we obtained a novel sampling optical temporal analyzer (SOTA) with high sensitivity. We were able to measure extremely weak signals with picosecond resolution.

We reported earlier ${ }^{4}$ that an interdigitated MSM photoconductive detector grown on low-temperature (LT) GaAs can function as either a high-sensitivity photodetector or photoswitch. As a detector, operating at a low input optical power level of $4 \mu \mathrm{W}(0.04 \mathrm{pJ} /$ pulse $)$, the device has a response time of $1.2 \mathrm{ps}$ and responsivity of $0.1 \mathrm{~A} / \mathrm{W}$. As input optical power level increases to $>1 \mathrm{~mW}(10 \mathrm{pJ} / \mathrm{pulse})$, the same device functions as high-sensitivity photoconductive switch. With an input power of $2.2 \mathrm{~mW}(22 \mathrm{pJ} / \mathrm{pulse})$, the gap resistance is driven from $10^{7}$ to $30 \Omega$, while the time resolution increases to only 1.5 ps. Our experiment takes advantage of this unique functionality for picosecond measurement of low-intensity optical signals.

Figure 1 shows the schematic diagram of the SOTA experiment. The interdigitated structure bridging between the coplanar transmission lines functions as the photodetector. We placed interdigitated detector between coplanar transmission lines instead of using photoconductive gap switching to assure good coupling of the generated electrical pulse to the propagating mode. The second interdigitated structure, connected between the ground electrode of the transmission lines and the sampling line, functions as photoswitch to

\footnotetext{
"Current address: AT\&T Bell Laboratories, Rm. 4H616, 101 Crawfords Comer Road, Holmdel, NJ 07733-3030.

b) Current address: Picotronix Inc., P.O. Box 130243, Ann Arbor, MI 48113-0243.
}

sample the signal. The separation between the photodetector and the photoswitch is $100 \mu \mathrm{m}$ in our case. The detector and switch are identical. The finger width and spacing are 0.2 $\mu \mathrm{m}$. The active area is $7 \mu \mathrm{m} \times 8 \mu \mathrm{m}$. The coplanar transmission lines have a strip width of $20 \mu \mathrm{m}$ and a separation of 20 $\mu \mathrm{m}$. The characteristic impedance is $90 \Omega$. The entire structure was fabricated on LT-GaAs. The LT-GaAs layer was grown on a (100) semi-insulating GaAs substrate which has a $0.4-\mu \mathrm{m}$ conventionally grown undoped buffer layer. The LT-GaAs layer was grown to a thickness of $1.5 \mu \mathrm{m}$ at a substrate temperature of $190{ }^{\circ} \mathrm{C}$ and subsequently annealed at $600^{\circ} \mathrm{C}$ for $10 \mathrm{~min}$ in an arsenic overpressure. The interdigitated electrodes are fabricated of $300 \AA / 2000 \AA \mathrm{Ti} / \mathrm{Au}$ using a JEOL JBX 5DIIF direct-write $e$-beam lithography system. Coplanar transmission line electrodes of $20-\mu \mathrm{m}$ spacing/width and 5-mm length are also fabricated of 500 $\AA / 2500 \AA \mathrm{Ti} / \mathrm{Au}$ using conventional optical lithography. No antireflection coating is placed over either the photodetector or the photoswitch in this initial work.

We use the photoconductive sampling technique to characterize the SOTA. A balanced, colliding pulse, mode-locked dye laser operating at $610 \mathrm{~nm}$ with a repetition rate of 100 $\mathrm{MHz}$ produces 150 fs pulses. We use 150 fs optical pulses for both the signal under study and the gate pulse. As shown in Fig. 1, the pump beam is focused onto the interdigitated photodetector and its average power adjusted from $4 \mathrm{~mW}$ to $1 \mathrm{nW}$ using a variable attenuator. The detector is dc-biased through the coplanar transmission lines. An electrical pulse is switched into the transmission lines as the femtosecond pump optical pulse hits the interdigitated photodetector. The signal is sampled at the photoconductive gate as the gate pulse turns on the photoswitch. The gate pulse typically has a power of $3 \mathrm{~mW}$ ( $30 \mathrm{pJ} / \mathrm{pulse}$ ). The current obtained at the sampling gate is amplified by a current amplifier.

Figure 2 shows the temporal response of the SOTA. A set of wave forms are measured at different input optical power levels. Each curve represents almost three orders of magnitude difference in average optical power. The highest power is $4 \mathrm{~mW}$ ( $40 \mathrm{pJ} /$ pulse), the lowest power being $8 \mathrm{nW}$ $\left(80 \times 10^{-6} \mathrm{pJ} /\right.$ pulse $)$, spanning almost six orders of magnitude. The dc bias on this $0.2 \mu \mathrm{m}$ interdigitated detector is 4 $V$. The dramatic change in pulse energy shows virtually no degradation in the response of the SOTA. The full width at half-maximum for input optical power of $4 \mu \mathrm{W}$ is $1.9 \mathrm{ps,}$ matching the convolution value of $1.9 \mathrm{ps}$ for detector (1.2 ps) and gate $(1.5 \mathrm{ps})$ responses. The response of the SOTA for 4 $\mathrm{mW}$ input power is slightly slower due to the slower re- 


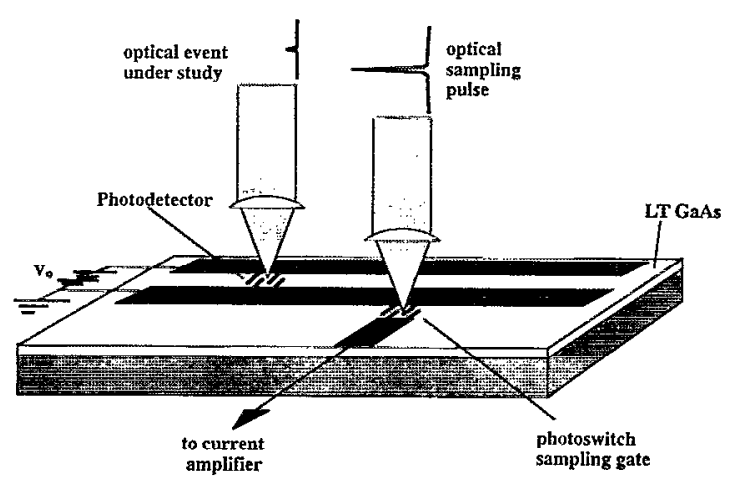

FIG. 1. Schematic diagram of the SOTA experiment.

sponse of the interdigitated photodetector at high input power levels. ${ }^{4}$ As input power decreases to $8 \mathrm{nW}$, the signalto-noise ratio is still 5:1 with an integration time of $100 \mathrm{~ms}$. This corresponds to a noise equivalent power (NEP) of 500 $\mathrm{pW}$ for the SOTA, or 16 photons/pulse at $100 \mathrm{MHz}$ repetition rate. The dynamic range is more than $60 \mathrm{~dB}$.

For comparison, we performed an experiment without the interdigitated photodetector and photoswitch in place. Such a measurement is essentially a conventional photoconductive sampling with $20-\mu \mathrm{m}$ gap photoconductive switch and $20-\mu \mathrm{m}$ gap photoconductive gate. Figure 3 shows the response for both conventional photoconductive sampling and the SOTA measurement. The input power for the conventional photoconductive sampling is $0.8 \mathrm{~mW}$, and the input power the SOTA is only $0.1 \mu \mathrm{W}$. However, the signalto-noise ratio is approximately equal for such dramatic change in input power level. This indicates that the SOTA is capable of detecting an optical signal 8000 times weaker. The 8000 times increase in sensitivity for the SOTA is the result of increased sensitivity of both detector and gate by using interdigitated structures. The switched peak voltage $V_{\text {out }}$ on the transmission lines is given by ${ }^{5}$

$$
V_{\text {out }}=V_{\text {dc }}\left[Z_{0} /\left(R_{s}+Z_{0}\right)\right] \text {, }
$$

where $V_{\mathrm{dc}}$ is the dc bias on the transmission lines, $Z_{0}$ is the

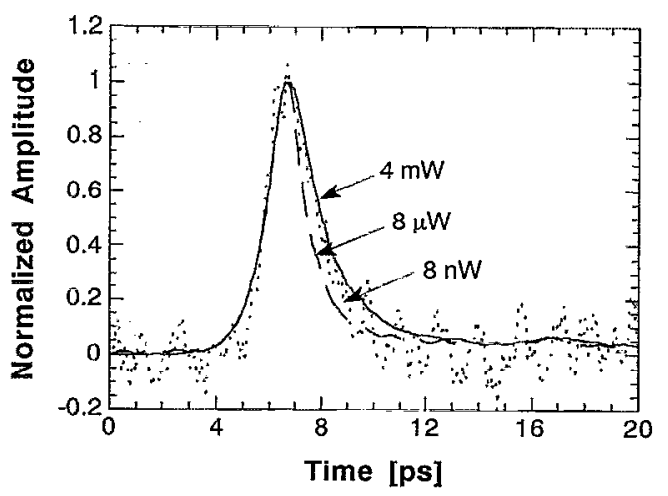

FIG. 2. Temporal response of the SOTA for different input power levels. The three curves in the figure represent the input power levels of $4 \mathrm{~mW}, 8$ $\mu \mathrm{W}$, and $8 \mathrm{nW}$, respectively, spanning almost six orders of magnitude.

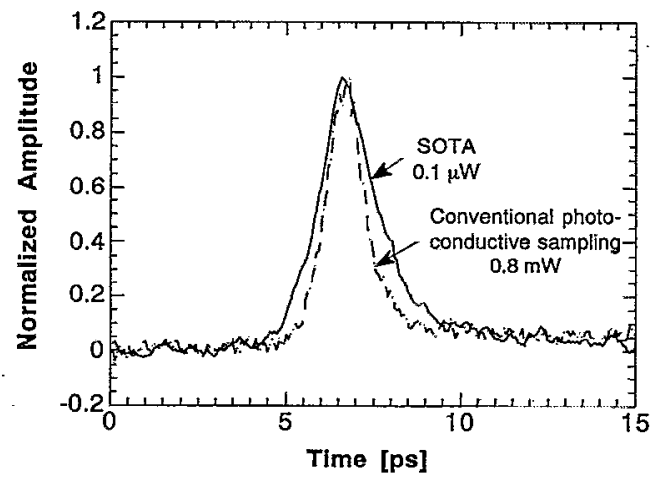

FIG. 3. Temporal response of the SOTA compared with a conventional photoconductive (PC) gap sampling. The two curves have approximately equal signal-to-noise ratio, though their input signals differ by a factor of 8000 .

characteristic impedance of the transmission lines, and $R_{s}$ is the on-state resistance of the photoconductive gap. The onstate resistance for a $20-\mu \mathrm{m}$ photoconductive gap is $5 \mathrm{k} \Omega$ as measured directly from electro-optic sampling. ${ }^{6}$ The on-state resistance of the interdigitated photodetector is only $30 \Omega$ for the same power level. ${ }^{4}$ If we assume every other condition is the same, the switched voltage for the SOTA at the detector end is 41 times higher than that of a simple coplanar transmission line switching as obtained from Eq. (1). At the gate, the switched current to current amplifier is at least 166 higher for the SOTA as a result of 166 reduction in photoconductive gate resistance. The combinational gain in sensitivity is at least $41 \times 166$, or 6833 . This is very close to what we obtained in the experiment. We also notice in Fig. 3 that the response of the conventional photoconductive sampling is faster than that of the SOTA response. This is the result of finite capacitancc of the interdigitated structures of the SOTA system.

We now analyze the noise sources that limit the NEP of the SOTA. There are noise contributions from both photodetector and photoswitch of the SOTA. The dominant noise currents are shot, $i_{\text {shot }}$, and thermal noise current, $i_{\text {thermal, }}$ given by the following equations, ${ }^{7}$ respectively:

$$
\begin{aligned}
& i_{\text {shot }}=\sqrt{2 q\left(I_{P}+I_{D}\right)}(\mathrm{A} / \sqrt{\mathrm{Hz}}), \\
& i_{\text {thermal }}-\sqrt{4 k T G}(\mathrm{~A} / \sqrt{H z}),
\end{aligned}
$$

where $q$ is the electron charge, $I_{P}$ is the average signal current, $I_{D}$ is the dark current, $k$ is the Boltzmann constant, $T$ is the temperature, and $G$ is the average conductance of the gate. At the detector, the average photocurrent $I_{P}$ is $2.5 \times 10^{-11} \mathrm{~A}$ with $500 \mathrm{pW}$ average optical power, obtained from $0.1 \mathrm{~A} / \mathrm{W}$ responsivity and taking into account of current going two directions. The dark current is $400 \mathrm{nA}$ in this case. The average thermal conductance is $10 \mathrm{M} \Omega$, obtained by the bias divided by the total average current. Using these parameters, we obtain $i_{\text {shot }}=1 \times 10^{-15} \mathrm{~A} / \mathrm{Hz}^{1 / 2}$ and $i_{\text {thermal }}=4 \times 10^{-14}$ $\mathrm{A} / \mathrm{Hz}^{1 / 2}$ from Eqs. (2) and (3), respectively, for the photodetector. The average photocurrent is several orders of magnitude above both the shot and thermal noise floor at the detector end. The measured signal current $I_{P}$ at photoconductive switch is $3.2 \times 10^{-13} \mathrm{~A}$ as obtained from 
current amplifier. The dark current is negligible. We calculate $i_{\text {shot }}=3.1 \times 10^{-16} \mathrm{~A}$ from Eq. (2). The off-state resistance is $10 \mathrm{M} \Omega$ and on-state resistance is $30 \Omega$ at the photogate. With a duty cycle of $1.5 \times 10^{-4}$ (1.5 ps gate open time versus $10 \mathrm{~ns}$ pulse repetition rate), this gives an average conductance of $5.1 \times 10^{-6}$ mhos. The correspondent thermal noise is $2.9 \times 10^{-13} \mathrm{~A} / \mathrm{Hz}^{1 / 2}$ as calculated from Eq. (3), far more dominant than shot noise at the gate. The thermal noise current amplitude is close to the signal current at gate. We conclude that the NEP of the SOTA system is limited by thermal noise at gate. We notice that only $1 / 80$ of the signal current on the transmission lines is switched to the current amplifier, even though the gate resistance at on state is lower than the transmission line impedance. At present, it is not completely clear to us what is limiting the switching efficiency of the gate.

In conclusion, we have demonstrated a sampling optical temporal analyzer with the integration of two interdigitated structures on coplanar transmission lines. The SOTA is able to measure extremely low optical signals with picosecond resolution and high dynamic range. The photoconductive sampling nature of the SOTA makes it also an effective tool for timing multiple optical events at picosecond resolution.
This enables us to perform ultraweak fluorescence and scattering experiments as well as optical ranging experiments that occur on the picosecond time scale.

The authors thank Frank Smith and Arthur Calawa for providing LT-GaAs samples. The work was supported by Army Research Office under Contract DAAL 03-89-K-0071, AFSC-Rome Laboratory under Contract F19628-90-K-0015, and the Air Force Office of Scientific Research (AFOSR) University Research Initiative under Contract 90-0214.

${ }^{1}$ D. H. Austin, Appl. Phys. Lett. 26, 102 (1975).

${ }^{2}$ K. D. Li, A. S. Hou, E. Ozbay, B. A. Auld, and D. M. Bloom, Appl. Phys. Lett. 61, 3104 (1992).

${ }^{3}$ M. B. Ketchen, D. Grischkowsky, T. C. Chen, C. C. Chi, I. N. Duling, N. J. Halas, J.-M. Halbout, J. A. Kash, and G. P. Li, Appl. Phys. Lett. 48, 753 (1986).

${ }^{4}$ Yi Chen, Steve Williamson, Tim Brock, F. W. Smith, and A. R. Calawa, Appl. Phys. Lett. 59, 1984 (1991).

${ }^{5}$ W. C. Nunnally and R. B. Hammond, Picosecond Optaelectranics Devices, edited by C. H. Lee (Academic, Orlando, Florida, 1984), p. 373398.

${ }^{6}$ M. Y. Frankel, J. F. Whitaker, G. A. Mourou, F. W. Smith, A. R. Calawa, IEEE Trans. Electron Devices ED-37, 2493 (1990).

${ }^{7}$ S. M. Sze, Physics of Semiconductor Devices, 2nd ed. (Wiley, New York, 1981), p. 111. 